\title{
Protection of power transformers against the effect of magnetic storms
}

\author{
Daniel Mayer ${ }^{1}$
}

\begin{abstract}
In the operation of large-scale power systems for the long-distance transmission of large amounts of electricity, a number of cases have been reported in which anomalies in the Earth's magnetosphere, referred to as geomagnetic storms, have caused a severe system collapse. Changes in the geomagnetic field cause a semi-saturating phenomenon, in which the high-voltage lines and especially the high-voltage windings of the power transformers of the system are overloaded with current and subsequently also thermally. The present article briefly explains the physical nature of magnetic storms and then describes a new device that either eliminates the possibility of a step-down power transformer accident or significantly reduces its effects on the system. The essence of this device are frequency filters, which are connected in parallel to the high-voltage windings of power transformers. At the beginning of a geomagnetic storm, the frequency filter is automatically connected to the system and is automatically disconnected when it subsides. The operation of frequency filters does not require human intervention, acquisition and operating costs are low and their integration into existing power systems is easy.
\end{abstract}

Keywords: geomagnetic field, coronal mass ejection, geomagnetically induced currents, magnetic semi-saturation, frequency filter

\section{Introduction}

At present, when human civilization is heavily dependent on electrical engineering and electronics, magnetic storms can severely disrupt the functioning of various electrical engineering systems. The more advanced technology a person uses, the more vulnerable this technique is, and thus the threat of dangerous magnetic storms becomes relevant. In addition to various advanced technical devices (eg satellite of telecommunications networks, navigation systems, etc.), magnetic storms can damage electrical systems for the transmission of electricity, especially power transformers, and cause large power outages.

\section{Geomagnetism and geomagnetic storms}

The geomagnetic field forms the Earth's magnetosphere [1-8]. The source of the geomagnetic field are both physical processes inside the Earth and physical processes in the ionosphere of the Sun. Thus, according to the source, we distinguish between internal and external geomagnetic fields; both magnetic fields are superimposed.

\subsection{Internal geomagnetic field}

The idea of the Earth as a permanent magnetic dipole (William Gilbert, 1600) was abandoned after the discovery that the Earth's core had a temperature above Curie's temperature. For example, at a depth of $100 \mathrm{~km}$ below the surface, the temperature is of the order of
$1000{ }^{\circ} \mathrm{C}$, ie substantially higher than the Curie temperature. Since 1919, a model called geodynamo has physically explained geomagnetism. According to this idea, the internal geomagnetic field is induced by the rotational flow of the Earth's liquid core. Since it is a moving electrically conductive medium that is exposed to a magnetic field (very weak, such as the Sun's magnetic field), very strong electric currents are induced in it, which generate a geomagnetic field. This relatively simple model was initially designed as stationary. However, he did not explain the well-known fact that the internal geomagnetic field changes with time - there is talk of secular variations. Secular variations are very slow, being detected on a scale of tens to thousands of years. Therefore, the original idea that the geomagnetic field is induced by axially symmetrical, stationary flow was abandoned and the geodynamic model was gradually improved. Modern geodynamic theory envisages a very complex model, respecting turbulent and non-stationary magnetohydrodynamic flow. This model has not yet been solved mathematically even with the help of powerful computers. However, an approximate solution was found, which well explains the secular variations and the possibilities of polarity reversal of the Earth's magnetic poles.

The intensity of the internal magnetic field varies with place on Earth. In our latitudes, the magnetic induction has a value of about $44 \mathrm{nT}$, at the poles around $60 \mathrm{nT}$ and at the magnetic equator around $30 \mathrm{nT}$. Due to the very slow time course of secular variations, the influence of the internal geomagnetic field on the electrical system is completely insignificant.

\footnotetext{
${ }^{1}$ Department of Electrical Engineering and Computer Modeling, University of West Bohemia, Plzen, Czech Republic, mayer@fel.zcu.cz
} 


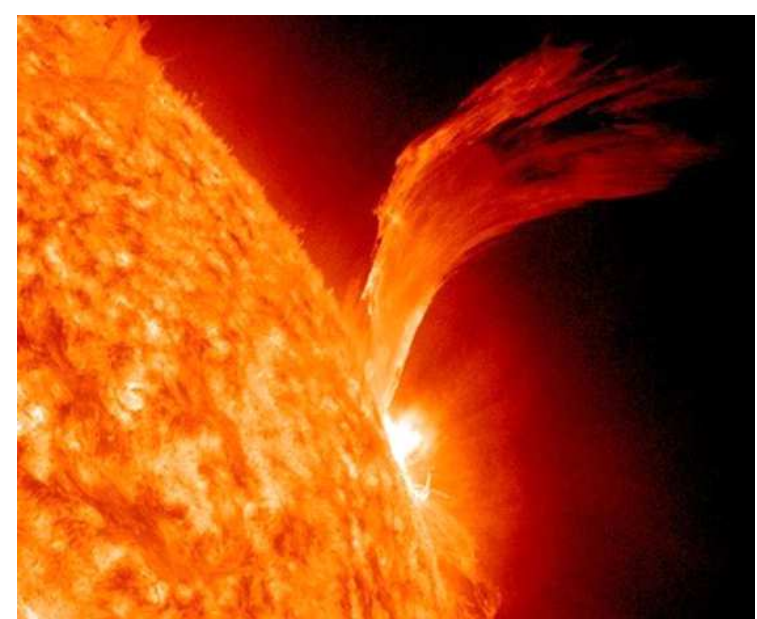

Fig. 1. Eruption at the edge of the solar disk observed by the STEREO satellite [4]

\subsection{External magnetic field}

There are relatively fast variations of the geomagnetic field, which have their origin in solar activity [1-4]. One of the manifestations of solar activity are solar flares, Fig. 1. These massive explosions produce intense electromagnetic radiation in a wide range of spectra and create jets of solar matter. Solar matter containing electrically charged particles, especially electrons, protons and high-energy alpha particles, can be torn off. They spread at high speed through interplanetary space and are referred to as coronal mass ejection (CMA). Iheir flow is called the solar wind. For the process of solar wind flow through interplanetary space, the designation Space weather has been adopted. If a wave of the solar wind comes close to the Earth, its internal geomagnetic field - the Earth's magnetosphere - prevents electrically charged particles from hitting the Earth. Under the action of the Lorentz force, the electrically charged particles of the solar wind move in the direction of the magnetic field lines of the magnetosphere, bypass the Earth, deform the original symmetrical shape of the internal geomagnetic field and flow further into interplanetary space. The Earth's magnetic envelope thus shields the Earth from the solar wind, thus protecting the Earth's biosphere. Without internal geomagnetism, there would be no existing forms of life on our planet. Some of the electrically charged particles of the solar wind penetrate into the higher layers of the Earth's atmosphere, ionosphere, turn to the Earth's magnetic poles and cause ionization of the Earth's atmosphere. With increased solar activity, the ionization is stronger and this process manifests itself as aurora borealis. The movement of electrically charged particles in the ionosphere represents an electric current that induces an external magnetic field in its vicinity. The waves of the solar wind then manifest on the Earth by rapid variations of the external magnetic field.

The intensity of the external magnetic field changes in the order of seconds or tens of seconds. Their ampli- tude varies: from small disturbances of 20 to $30 \mathrm{nT}$ and occurring several times a day, to strong variations whose amplitudes can reach up to hundreds of $\mathrm{nT}$ then there is talk of a geomagnetic storm. It usually takes two to four days between a solar flare and a magnetic storm on Earth. The frequency and intensity of magnetic storms depends on the Earth's position; they are higher near the earth's magnetic poles, $i e$ in the Nordic countries. A geomagnetic storms cannot be influenced or even averted by human possibilities, but a few hours before it affects the Earth, its intensity can be predicted and the area on Earth affected by the storm can be determined approximately. Next, we describe the device that can prevent the destructive effects of magnetic storms on power systems.

\subsection{Geomagnetic field monitoring}

A worldwide network of geomagnetic observatories monitors the geomagnetic field [9]. In the Czech Republic, the geophysical observatory is located in the village of Budkov near Prachatic and is one of the workplaces of the Geophysical Institute of the Academy of Sciences of the Czech Republic. All three components of the geomagnetic induction vector $\left(B_{x}, B_{y}, B_{z}\right)$ are measured continuously here and the results are recorded, at $1 \mathrm{~min}$ intervals or at second intervals, respectively, with an accuracy of $0.1 \mathrm{nT}$. The results are then passed on to the World Data Center in Boulder (USA) and the Edinbourgh Geomagnetic Information Node (Scotland). The Space Weather Prediction Center (SWPC) publishes a three-day space weather forecast. The results are freely available on the Internet. In addition to continuous monitoring of the geomagnetic field, solar activity is constantly monitored [3], [10]. In addition to observing solar flares with solar telescopes, these phenomena are continuously monitored by satellite (for example, SOHO and STEREO satellites) by the US National Oceanic and Atmospheric Administration (NOAA), Boulder and the European Space Agency (ESA) Paris. At the moment of detection of coronary matter heading to Earth, a mathematical calculation is performed at the Goddard Space Flight Center (GSCF) to determine the density at which it will hit the Earth's magnetosphere and then predict the intensity and location of the magnetic storm on Earth. This information is then passed on to the power system operators.

\section{Geomagnetic induced currents (GIC)}

The geomagnetic field $\boldsymbol{B}(t)$ acts on the earth's crust and on metal structures placed above the earth's surface, $e g$ on outdoor power lines. In this environment, an electric field $\boldsymbol{E}$ is induced, and because it is an electrically conductive environment (with conductivity $\gamma$ ), geomagnetically induced currents (GIC) of density $\boldsymbol{J}$ flow through it. According to the law of electromagnetic induction and Ohm's law is valid

$$
\operatorname{rot} \boldsymbol{E}=-\frac{\partial \boldsymbol{B}}{\partial t}, \quad \boldsymbol{J}=\gamma \boldsymbol{E} .
$$




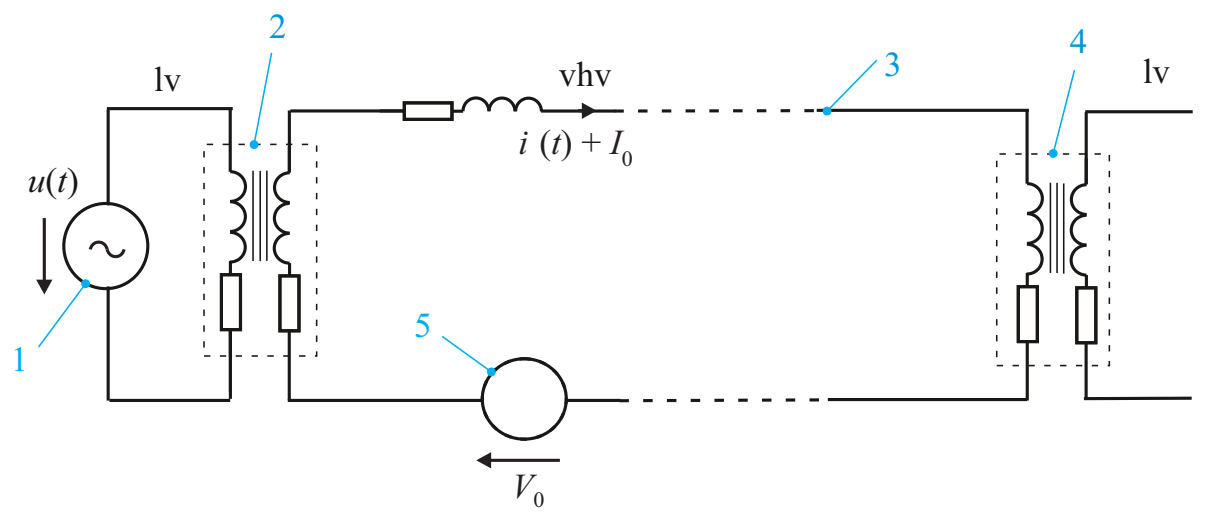

Fig. 2. Simple model of single-phase electrical supply system: 1 - generator, 2 - step-up power transformer, 3 - very high voltage transmission line, 4 - step-down power transformer, 5 - source of quasi-variable voltage induced by magnetic storm

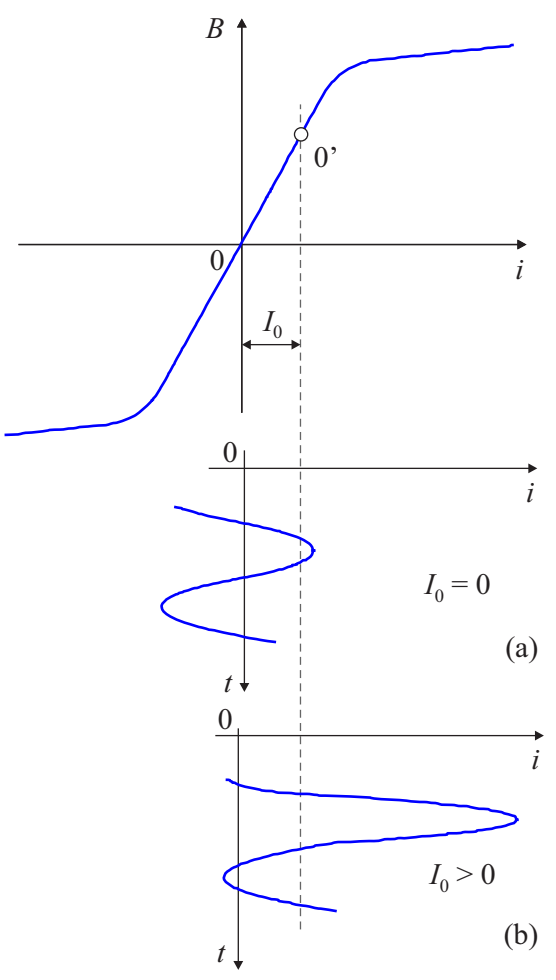

Fig. 3. Influence of supersaturation of the magnetic circuit of the power transformer on the occurrence of current overload the phenomenon of semisaturation of the magnetic circuit of the power transformer: (a) - without supersaturation, (b) - with supersaturation

During magnetic storms, GICs reach high values and cause accidents of power transformers. From (1) it follows an important finding that the magnitude of GIC depends on the derivation of the geomagnetic induction vector $\boldsymbol{B}(t)$, ie on the rate of variation of the external geomagnetic field, not on its magnitude, as is often mistakenly stated.

The magnetic induction $\boldsymbol{B}(t)$ and thus also the GIC have a random time course, and although there is talk of rapid variations in the external magnetic field, it changes relatively slowly, compared to alternating electrical quantities of industrial frequency. Magnetic storms are changes in units of $\mathrm{nT} / \mathrm{min}$. Depending on the time, GICs es- sentially behave as direct current currents, we call them quasi-stationary. Only their ohmic resistances determine the distribution of GIC in the conductors of the power system. Inductances and capacitances of the electrical network do not affect the GIC. In works $[7,8]$ a method of determining the distribution of GIC in a general power system is formulated, which can be topologically arbitrarily complex.

\section{Effect of magnetic storm on unprotected power system}

The essence of the destructive effect of a magnetic storm on the power system is explained using a simple single-phase model of the system according to Fig. 2. A source of harmonic voltage (generator) $u(t)$ of frequency $f$ supplies electrical power to the system a long line of vhv to the place of consumption, where vhv/lv is transformed and supplies the distribution network, from which electricity is taken by individual consumers. In Fig. 2, a load consisting of a resistor and an inductance represents the distribution network. A magnetic storm characterized by magnetic induction $\boldsymbol{B}(t)$ acts on this electrical system. Vector $\boldsymbol{B}$ is a superposition of the internal magnetic field (it does not change with time) and the external magnetic field (it changes "slowly" with time, it is quasistationary). Only its vertical component $B_{z}(t)$ is applied, $i e$ the component perpendicular to the drawing in Fig. 2. In the loop formed by the high-voltage line, which is connected at its beginning and end to the high-voltage windings of the power transformers is induced (according to the law of induction) a voltage

$$
V_{0}(t)=\frac{\mathrm{d} \Phi}{\mathrm{d} t}=S \frac{\mathrm{d} B_{z}}{\mathrm{~d} t} .
$$

This equation holds under the assumption that intensity of the geomagnetic field $B_{z}$ is equally distributed along the high-voltage line. In (2), the magnetic induction flux $\Phi$ is linked with a loop whose area is $S=a l$, where $l$ is the length of the vhv line and $a$ is the distance between the conductors of the high voltage line. 


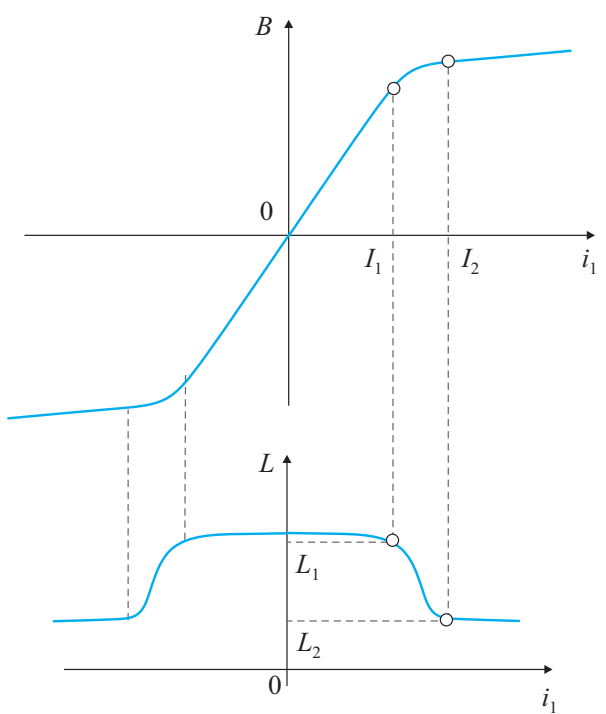

Fig. 4. The course of inductance of the power transformer winding during supersaturation of its magnetic circuit

The induced voltage $V_{0}$ in the loop results in an induced GIC $I_{0}$. The internal geomagnetic field is stationary, ie according to (2) it does not contribute to the magnitude of the induced voltage $V_{0}$ (ie not even to GIC $I_{0}$ ). In contrast, the external geomagnetic field changes over time, but "slowly" (compared to the current supplied by the generator $i(t)$, whose frequency is $50 \mathrm{~Hz}$ ), is quasistationary. Only the resistances in this loop limit the magnitude of the current $I_{0}$ in the loop, formed by the vhv line and the high-voltage windings of both transformers. Measurements on real power systems have shown that the induced voltage $V_{0}$ reaches in the order of up to hundreds of $\mathrm{V}$ during magnetic storms and the subsequent current $I_{0}$ in the vhv line up to tens of A [11],[18].

Geomagnetically induced current $I_{0}$ is superimposed to the working current $i(t)$ supplied by the source, the current $I_{0}+i(t)$ passes in the vhv line. Its rms value is a measure of dissipated thermal energy. (According to Joule's law, the dissipated electrical energy per thermal energy is proportional to the square of the rms current value.) This energy thermally endangers the conductors of the high-voltage line, but above all the high-voltage windings of both transformers. The high-voltage line is designed as a bundle conductors and is dimensioned in such a way that there is no risk of thermal damage. It is more complicated with transformers. Let us observe the current $i(t)$ in a high voltage line.

- If there is no magnetic storm, GIC $I_{0}=0$ and both transformers operate in normal mode. The operating point moves in the linear part of their magnetization characteristic, ie below the knee of this curve. The power system according to Fig. 2 is linear, all currents and voltages in the circuit change harmoniously, Fig. 3(a).

- During a magnetic storm, a quasi-stationary voltage $V_{0}$ is induced in the high-voltage line and a quasistationary GIC $I_{0}$ passes through the line. Let us observe the current flowing through the vhv line. The operating point on the magnetization curve of the transformers is shifted by the value $I_{0}$, the zero position of the operating point is 0 , Fig. 3(b). In the half-period, when $i(t)<I_{0}$ the operating point moves in the linear part of the magnetization curve (ie below the knee), the system is linear and current overload of the transformers does not occur. However, in the half-period, when $i(t)>I_{0}$ the operating point passes to the nonlinear part of the magnetization curve (ie at and above the knee) and the current $i(t)$ supersaturates the magnetic circuits of the transformers. The permeability of magnetic circuits $(\mu=B / H)$ has decreased and thus the inductance of the transformer windings will also decrease, the system is nonlinear, Fig. (4). Due to the quasi-stationary current $I_{0}$, the magnetizing current curve has changed its shape, its effective value is considerably higher than when the geomagnetic field did not affect the system, $i e$ when it was $I_{0}=0$, the highvoltage windings of both transformers become overheated. This phenomenon is called semisaturation of magnetic circuits. The theoretical solution [5,6] (and the investigation of faults in practice [12] to [19] (show that the current overload can range from 2.5 times to three times the nominal value.

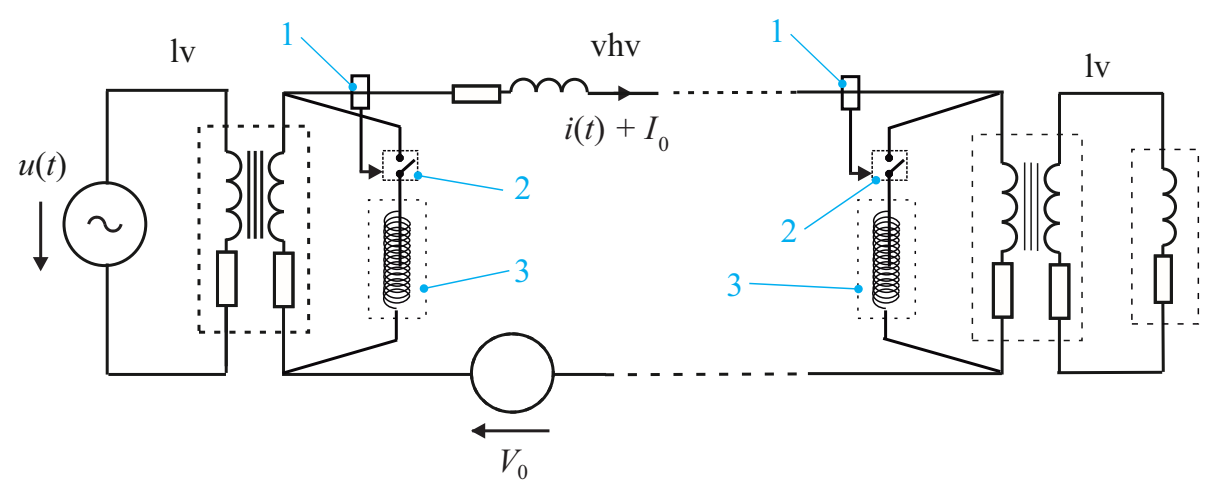

Fig. 5. A simple model of a single-phase electrical supply system, whose power transformers are protected by superconducting reactors: 1 - sensor-indicator of the quasi-stationar current $I_{0}$ in the high voltage transmission line, 2 - a switch that connects/disconnects the superconducting reactor to the high voltage winding of the power transformer, 3 - single-phase superconducting reactor 


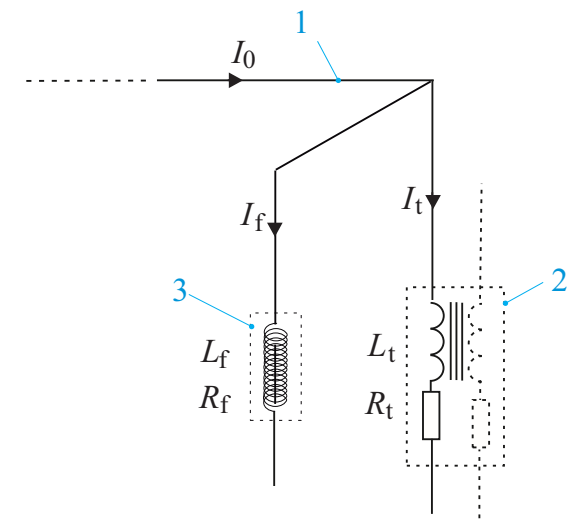

Fig. 6. Distribution of the quasi-direct current $I_{0}$ into the current $I_{\mathrm{f}}$ passing through the frequency filter 3 and into the current $I_{\mathrm{t}}$ in the high-voltage winding of the transformer 2

\section{A new way of protecting transformer windings from magnetic storms}

A new method of protecting the transformer windings consists in connecting a frequency filter in parallel with the high-voltage winding of both transformers during a geomagnetic storm, Fig. 5. The frequency filter consists of a coil wound on a ferromagnetic core. It can be modeled by series connection of resistance $R_{\mathrm{f}}$ and inductance $L_{\mathrm{f}}$, Fig. 6. The current $I_{0}$ induced in the vhv line is divided into the current $I_{\mathrm{f}}$ passing through the frequency filter and the current $I_{\mathrm{t}}$ in the high-voltage winding of the transformer

$$
I_{\mathrm{f}}=\frac{R_{\mathrm{t}}}{R_{\mathrm{f}}+R_{\mathrm{t}}}, \quad I_{\mathrm{t}}=\frac{R_{\mathrm{f}}}{R_{\mathrm{f}}+R_{\mathrm{t}}},
$$

All three currents are quasi-stationary, so limited only by resistances.

- A frequency filter with zero resistance $\left(R_{\mathrm{f}} \rightarrow 0\right)$ will provide perfect protection for the transformer. According to (3), all current $I_{0}$ then flows through the frequency filter $\left(I_{\mathrm{f}}=I_{0}\right)$, while no GIC passes through the windings of the transformers $\left(I_{\mathrm{t}}=0\right)$. Such a frequency filter can be realized by a coil (with a ferromagnetic core), made of a high-temperature superconductor, brought into a superconducting state. It is a superconducting reactor, which includes a cryotechnical device that produces liquid nitrogen, which cools the frequency filter coil to a critical temperature at which the coil resistance is $R_{\mathrm{f}} \rightarrow 0$. The advantage of this frequency filter is perfect protection of the transformer winding, the disadvantage is the need cryotechnical equipment.

Note that superconducting coils are usually used to generate extremely strong magnetic fields, using extremely high magnetizing currents. Thus, non-ferrous ("air") superconducting coils are used for strong magnetic fields. In our case, however, the superconductor of the frequency filter coil only serves to reach $R_{\mathrm{f}}=0$, so the coil can have a magnetic circuit made of ferromagnetic material, which is of course advantageous.
- If the coil of the frequency filter is made of a common (not superconducting) material, for example from copper, $R_{\mathrm{f}} \neq 0$. The filter provides only partial protection. (For example, when $R_{\mathrm{f}}=R_{\mathrm{t}}$, then $I_{\mathrm{t}}=0.5 I_{0}$.) For partial protection of the transformers, a frequency filter can be implemented with a conventional iron reactor. The sharper the inequality $R_{\mathrm{f}}<R_{\mathrm{t}}$, the more efficient the protection by the frequency filter, but the greater the weight and dimensions of the reactor. The advantage of this method of transformer protection is that it does not require cryotechnical equipment, the disadvantage is only partial protection. As an example, consider a frequency filter whose resistance is $R_{\mathrm{f}}=R_{\mathrm{t}} / 4$, ie the cross-section of its coil is four times larger than the cross-section of the high voltage winding of the transformer. Then, according to (3), a quasistationary current in hv winding of the transformer is $I_{\mathrm{t}}=0.2 I_{0}$, ie only 20 percent of current $I_{0}$ in the hv winding of the transformer in comparison with the current in the unprotected winding. However, at the cost of the frequency filter having approximately four times the weight of the transformer's hv winding.

The connection/disconnection of the frequency filter to the power system can be automated, Fig. 5 . If a geomagnetic storm $B_{z}(t)$ acts on the power system, a quasistationary voltage $V_{0}$ and then a current $I_{0}$ is induced in the vhv line. Its magnitude is monitored by sensor 1. As soon as the current $I_{0}$ reaches a preselected (adjustable) value, it activates both switches 2 at the beginning and end of the transmission path and connects frequency filters in parallel to the high-voltage windings of both transformers. The current $I_{0}$, in whole or in part (depending on the type of frequency filter), does not flow through the high-voltage windings of both transformers, but through frequency filters. As soon as the geomagnetic storm stops, the current $I_{0}$ drops and the sensor activates both switches 2 , which disconnect both frequency filters from the transformer windings. In addition to the quasi-stationary current $I_{0}$, an alternating current also flows through the frequency filters, which has an inductive character and the impedance of the filter coil determines its magnitude. If this current increases the reactive power in the system and thus reduces the power factor $(\cos \varphi)$ to undesirable values, it can be compensated in the usual way, ie by connecting a compensator in parallel, which is most often a static capacitor.

Figure 7 shows a three-phase network with transformer protection by frequency filters at the point of connection to the distribution network. The three-phase superconducting reactor in connection $\mathrm{Y}$ is here connected to the high-voltage winding of a step-down power three-phase transformer, connected between the high-voltage transmission line and the distribution network. The transformer winding is connected to the grounded Y-D node. Similarly, at the point of connection of the generator to the step-up transformer, a superconducting reactor is connected in parallel to the high-voltage winding of the transformer. 


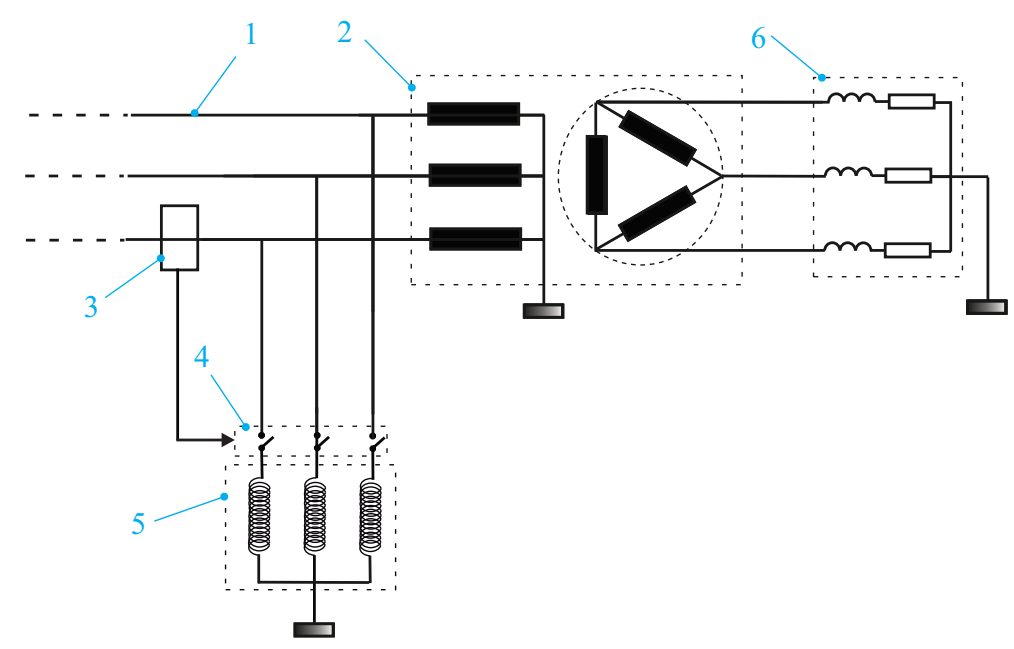

Fig. 7. Connection of a three-phase superconducting reactor to the high-voltage side of a step-down power transformer in a YD connection, converting electrical power into the distribution network: 1 - high voltage transmission line, 2 - three-phase step-down power transformer in YD connection with zero output, 3 - indicator of quasi-stationar current $I_{0}$ in high voltage transmission line, 4 switch to connect/disconnect superconducting reactor in the transmission system, 5 - three-phase superconducting reactor in connection $\mathrm{Y}$ with output zero, 6 - three-phase distribution network simulated by three-phase impedance in connection $\mathrm{Y}$ with ground

The essence of the new method of protection of transformers against the effects of magnetic storms was described here on an elementary model of the power system. However, frequency filters for complete or partial protection of transformers can be used in cooperation with power transformers that are part of any topologically complex system.

\section{Older proposals on how to protect the energy system from magnetic storms}

The methods of protection of power transformers against magnetic storms proposed so far are generally imperfect. For completeness, we will remind them here.

- Due to the predicted geomagnetic storm, a smaller or larger part of the system is switched off, which leads to a controlled collapse of the system. After the storm subsides, the system is restored to its original state. The disadvantage of this method of protection is the breach of supplier-customer relations between the provider and the consumer and, as a rule, the resulting need to compensate for economic losses caused by a power outage.

- The phenomenon of semisaturation, which is the cause of thermal overload and subsequent damage to transformers, develops only to a lesser extent in power transformers whose magnetic circuit is strongly oversized. In such robust transformers, the working area of the transformer lies in the linear part of its magnetization characteristic during DC pre-magnetization. The disadvantage is that the oversized, robust transformer is magnetically unused in normal operation. The robust transformer has more weight and dimensions, more losses in iron and more noise. Due to the rare occurrence of magnetic storms, its positive properties are used only sporadically and it is significantly uneconomical in terms of investment and operation.

Works [20-23] describe a power electronic device that limits a quasi-stationary GIC induced by a geomagnetic storm to a three-phase vhv line by conducting it to ground and thereby limiting its input to the hv winding of the power transformer.

\section{Conclusion}

The collapse of the electricity system is a serious technical event, which, especially in densely populated areas, can lead not only to huge economic losses, but also to threats to the safety of the population. One of the common causes of the collapse of the power system are rapid anomalies of the geomagnetic field - magnetic storms. Increasing industrialization means that disturbances caused by magnetic storms are not uncommon, especially in the Nordic countries, and have therefore recently received attention.

The presented work describes a new method of transformer protection, which uses frequency filters. Two types of frequency filters are described: filters with superconductor coils for complete protection, and filters with coils of common materials for partial protection. It is characterized by reliable exclusion, resp. limiting the occurrence of current overload of transformers, low purchase price, the possibility of easy adaptation to existing electrical systems and then automatic, unattended operation. The device is relatively simple and robust, which virtually eliminates its failure rate. 


\section{REFERENCES}

[1] E. N. Parker, Cosmical magnetic fields, Oxford, Clarendon Press, 1979.

[2] E. Priest, Solar magnetohydrodynamics, Dordecht, D. Reidel Publ. Co., 1984.

[3] R. E. Benestand, Solar aktivity and Earths climate, Berlin, Springer-Verlag, 2003.

[4] http://www.asu.cas.cz/cz/veda-a-vyzkum/vedecka-oddeleni/ slunecni-oddeleni, (In Czech).

[5] D. Mayer, "Geomagnetic storms and their effect on the electrical system.", ELEKTRO, no. 8-9, 2016, pp. 58-62,(In Czech).

[6] D. Mayer and Ulrych B. "Influence of magnetic storm on power system transformers.", ELEKTRO, no. 3, 2018, pp. 2-4, (In Czech).

[7] D. Mayer, "Contribution to investigation of influence of geomagnetic stroms on electrification system.", Acta Technica, 58 (2013), pp. 351-365.

[8] D. Mayer, "The effect of geomagnetic storms on the electrification system", Cyclonic and Geomagnetic Storms, Predicting factors, formation and enviromental impacts, Editor, Victoria P. Banks, Nova Science Publishers, Inc., New York 2015, pp. 1-18. ISBN 978-1-63482-360-9.

[9] https://www.ig.cas.cz/vyzkum-a-vyuka/oddeleni/geomagnetika /predpoved-geomagnetick-aktivity/, (in Czech).

[10] L. J. Lanzerotti, "', Space weather effects on technologies, Space weather effects on technologies, In, Song P. et al (eds.), Space Wheater. American Geophysical Union, Geophys. Monograph., 2001, 125, pp. 11-25.

[11] R. Nishiura, S. Yamashita, and S. Kano, "Simulation analysis of geomagnetically-induced currents (GIC) effects on shell-form transformers.", Power and Energy Society General Meeting, (PES), 2013 IEEE, 21. - 25. July 2013.

[12] R. Girgis, "Effects of geomagnetically induced currents on power transformers and power systems", CIGRE, 21, rue d' Artois, F-75008 Paris, A2-304, pp. 1-8.

[13] K. J. Patel, "An alalytic review of geomagnetically induced current effects in power system", Internat. Conf. On Electrical, Electronics and Optimization Techniques, (ICEEOT), 3-5 March 2016.

[14] J. R. Niño, "Core saturation effect of geomagnetic induced currents in power transformers", Journal of Applied Research and Technology, 14 (2016), pp. 87-92, 2011.

[15] F. Bachinger et al, "Direct current in transformers, effects and compensation.", CIGRE, //www.cigre.org).
[16] M. Stork and D. Mayer, "Direct currents in power transformers", Journal of Electrical Engineering, vol. 70 (2019), no. 1, pp. 68-72.

[17] J. Ramírez-Niño "Core saturation effect of geomagnetic induced currents in power transforms", Journal of Applied Research and Technology, 14 (2016), pp. 87-92.

[18] R. Price, "Geomagnetically induced currents effects on transformers", IEEE Transactions on Power Delivery, vol. 17, no. 4, October 2003, pp. 1002-1008.

[19] L. Bolduc, et al, "Development of a DC current-blocking dvice for transformer neutrals", IEEE Trans. on Power Delivery, 2995, Jan., vol. 20, no. 1, pp. 163-168.

[20] V. Gurevich, "Protection of power transformers against geomagnetically induced currents", Serbian Journal of Electrical Engineering, vol. 8, no. 2, November 2011, pp. 333-339.

[21] V. I. Gurevich, "Stability of microprocessor relay protection and automation systems against intentional descructive electromagntic impacts, Part I", Components and Technologies, no. 4, 2011, pp. 116-122. Part II, dtto, no. 5, 2011, pp. 129-136 (in Russian).

[22] V. I. Gurevich, Protection devices and systems for high-voltage applications, Marcel Dekker, New York, USA, 2003.

[23] H. Zhu and T. J. Overbyte, "Blocking device placement for mitigating of geomagnetically induced currents", IWWWE Trans. on Power Systems, 2015, July, vol. 30, no. 4.

[24] A. Rezaei-Zare, et al, "Analysis of three-phase transformer response due to GIC using an advanced duality-based model", Browse Journals and Magazines, IEEE Transactions on Power Delivery, vol. 31, no. 5, view document 273.

Received 5 August 20221

Daniel Mayer (Prof, Ing, DrSc) received the Ing, PhD and DrSc degrees in electrical engineering from Technical University in Prague, Czech Republic. In 1959 Associate Professor at the University of West Bohemia in Pilsen, in 1968 full Professor of the Theory of Electrical Engineering. Many years he was head of the Department of Theory of Electrical Engineering. Research interests: circuit theory, electromagnetic field theory, electrical machines and apparatus, history of electrical engineering. He published 9 books, more than 350 scientific papers and 14 patents. He is a member of editorial boards of several international journals and leader of many grant projects. 